\title{
A Social Force Model for Pedestrians' Movements Affected by Smoke Spreading
}

\author{
J. Makmul (iD \\ Department of Mathematics, Science Faculty, Kasetsart University, Thailand \\ Correspondence should be addressed to J. Makmul; fscijtm@ku.ac.th
}

Received 13 September 2020; Accepted 27 October 2020; Published 16 November 2020

Academic Editor: Agostino Bruzzone

Copyright () $2020 \mathrm{~J}$. Makmul. This is an open access article distributed under the Creative Commons Attribution License, which permits unrestricted use, distribution, and reproduction in any medium, provided the original work is properly cited.

\begin{abstract}
A social force (SF) model is proposed to simulate the egress of pedestrians while smoke is spreading. The advection-diffusion with source term is used to describe the propagation of smoke. It is incorporated into the SF model. The navigation field in our model is determined by the negative gradient of the solution of the Eikonal equation. It depends on the pedestrian and smoke density. Numerical experiments are performed in a room with multiple exits, and their results are shown.
\end{abstract}

\section{Introduction}

Many crowd disasters occurred all around the world. This leads to a large number of losses in human life and wealth. Due to these losses, many researchers study pedestrians' movements and their behaviors in a crowd, especially in evacuation situations. The development of pedestrian dynamic models can help to plan public buildings with respect to safety and evacuation. Nowadays, there are numerous pedestrian models, such as the particle flows [1], the social force model [2], the optimal-velocity model [3], the magnetic force model [4], the cellular automata models [5], and the discrete choice model [6].

There is quite a large number of researches focusing on modeling pedestrians' movements during smoke or fire spreading. Zheng et al. [7] proposed an extended floor-field model to study the influence of fire and smoke on the movement of pedestrians. The results of their simulations demonstrate that the fire location, the spreading rates of the fire, and the smoke highly influence the pedestrian evacuation. Weifeng and Hai simulated the crowd behavior in a smoke-filled compartment [8]. The pedestrians' visibility is affected by the smoke concentration in their model. Makmul [5] presented a cellular automaton (CA) model to simulate a pedestrian evacuation while gaseous hazardous material is spreading. It is combined with the advection-diffusion equation and the Eikonal equation. The advection-diffusion is used for hazard gas spreading. The Eikonal equation is applied for the arrival times of CA cells.

In this work, we are interested in the evacuation of pedestrians during the spread of smoke. A social force model is applied to simulate pedestrian movements, which is one of the most popular choices to model the dynamic behavior of pedestrians. This model produces realistic movements in simulations by taking the individual characteristics into account, such as the desired speed, desired destination, and physical interaction factors. In our model, the social force model is combined with the advection-diffusion equation, which is applied to the smoke density. This equation is used in many applications for fluid motion, heat transfer, flow of gas, or pollutant [9]. The operator splitting method is applied to solve this equation, which is an efficient approach to solve problems in multidimensions. The movement directions of an individual are defined by the negative gradient of the solution of the Eikonal equation in our model. The Eikonal equation is also incorporated into the SF model and solved numerically by the fast marching method [10].

Our main objective of this paper is to comprise the advection-diffusion equation [9] and the Eikonal equation [11] into a social force model. The expanded model is used 
to describe the pedestrian flow while smoke is propagating. The movement direction of a pedestrian is determined by the negative gradient of the solution of the Eikonal equation. The solution of the Eikonal equation depends on the smoke density as well as on the pedestrian density in the individual's visibility distance. The range of an individual's visibility varies all the time relying on the smoke density. We perform numerical experiments in a room with multiple exits. The influence of the pedestrian density and the smoke source location on the evacuation time is examined and discussed.

The framework of this paper is organized as follows. In Section 2, we present the social force model and explain a way to couple it with the advection-diffusion and the Eikonal equation. The numerical methods, which are used to approximate the solutions of the social force model, the advectiondiffusion equation, and the Eikonal equation are demonstrated in Section 3. Numerical experiments and results are shown in Section 4. Finally, the discussion and conclusions are made in Section 5.

\section{Model}

We study pedestrian evacuations in a multiple exits domain with a source of smoke. All pedestrians in our model are assumed to be familiar with the environment, and the smoke density has no effect on the pedestrians' health. A microscopic social force model without boundary force [2] is introduced to describe the pedestrians' movements. The moving direction of an individual in our model is guided by the solution of the Eikonal equation, which relies on the smoke density as well as on the pedestrian density in the visibility distance. The linear advection-diffusion equation is applied for the propagation of smoke. The microscopic social force equations of the dynamic interacting particle systems together with the Eikonal equation and the advection-diffusion equation in two dimensions read as

$$
\begin{gathered}
\frac{d x_{i}}{d t}=v_{i}(t), \\
m_{i} \frac{d v_{i}}{d t}=f_{i}^{d}(t)+\sum_{j \neq i}\left(f_{i j}^{\mathrm{soc}}(t)+f_{i j}^{\mathrm{ph}}(t)\right)+\xi_{i}(t),
\end{gathered}
$$

with location $x_{i} \in \mathbb{R}^{2}$ and velocity $v_{i} \in \mathbb{R}^{2}, i=1,2, \cdots, N$. $N$ is the number of all pedestrians. $m_{i}$ is the mass of pedestrian $i$, and $\xi_{i}(t)$ is an individual fluctuation term indicating chaotic behavioral changes. For simplification, we set $m_{i}=1$ and $\xi_{i}=0$ for all $i=1, \cdots, N$.

$f_{i}^{d}(t)$ is the desire force that indicates the intention of a pedestrian to reach his destination with a certain desired speed $v^{d}$ in a given desired direction $e^{d}$. Its mathematical expression for pedestrian $i$ is described by

$$
f_{i}^{d}(t)=\frac{v_{i}^{d}(t) e_{i}^{d}(t)-v_{i}(t)}{\tau_{i}}
$$

where $v_{i}^{d}(t)$ is the intended speed of pedestrian $i$ at time $t$. In our model, the intended speed of a pedestrian in a smoke region depends on the pedestrian density in the visibility distance. It is set to

$$
\begin{aligned}
& v_{i}^{d}(t)=U_{\max }\left(1-\frac{\rho\left(x_{i}, t\right)}{\rho_{\max }}\right), \\
& \rho\left(x_{i}, t\right)=\frac{1}{\pi\left(S^{t}\right)^{2}} \sum_{j,\left|x_{i}-x_{j}\right|<S^{t}} 1,
\end{aligned}
$$

$U_{\max }$ and $\rho_{\max }$ are the maximum speed and the density of pedestrians, respectively. $S^{t}$ is the visibility distance of a pedestrian in smoke areas at time $t . \rho(x, t)$ is the pedestrian density in a circle with s radius $S^{t}$ at time $t$. Experiments on human behavior in fire smoke show that the actual visibility distance captured by light reflecting objects can be described through the following equation $[7,12]$

$$
\begin{gathered}
S^{t}=\frac{3}{K^{t}}, \\
K^{t}=K_{p} m_{s}^{t},
\end{gathered}
$$

$K^{t}$ is the extinction coefficient of smoke at time $t$, which is measured in $m^{-1}$. $K_{p}$ is a constant, and it is set to $7.6 \mathrm{~m}^{2} / \mathrm{g}$ for light-reflecting objects such as human bodies. $m_{s}^{t}$ is the smoke mass at time $t$.

$e_{i}^{d}(t)$ is the unit vector pointing to the desired direction. $v_{i}(t)$ is the actual velocity, and $\tau_{i}$ is the relaxation time in which the pedestrian adapts his actual velocity to the intended velocity $\left[v_{i}^{d}(t) e_{i}^{d}(t)\right]$. In our model, the pedestrians intend to minimize their estimated travel time towards their destination but temper this behavior to avoid areas with high pedestrian and smoke densities. The desired movement direction of pedestrian $i$ in our model is given by

$$
e_{i}^{d}(t)=-\frac{\nabla T\left(x_{i}\right)}{\left|\nabla T\left(x_{i}\right)\right|},
$$

where $T\left(x_{i}\right)$ is the travel cost of the pedestrians to reach their destination at point $x_{i}$. It is the solution of the following Eikonal equation [11]

$$
|\nabla T(x)|=\frac{1}{F(x)}, \quad x \in \Omega,
$$

where $\Omega$ is a simulation domain and $T(x)$ is the arrival time of the front crossing the point $x . T(x)$ is set to 0 for the destination areas. $F(x)>0$ is the moving speed of the front and depends on the position of $x$. It is set as the follows

$$
F(x)=\left(\begin{array}{ll}
0.01 & , x \in \Omega_{b} \\
U(\rho(x)), & , x \in \Omega / \Omega_{b},
\end{array}\right.
$$

where $\Omega_{b}$ are areas that are obstructed by obstacles [11] or areas with high smoke density. We set the threshold smoke concentration as $C(x) \geq 0.05$. It is assumed to be high enough to influence the movements of the pedestrians. This value 


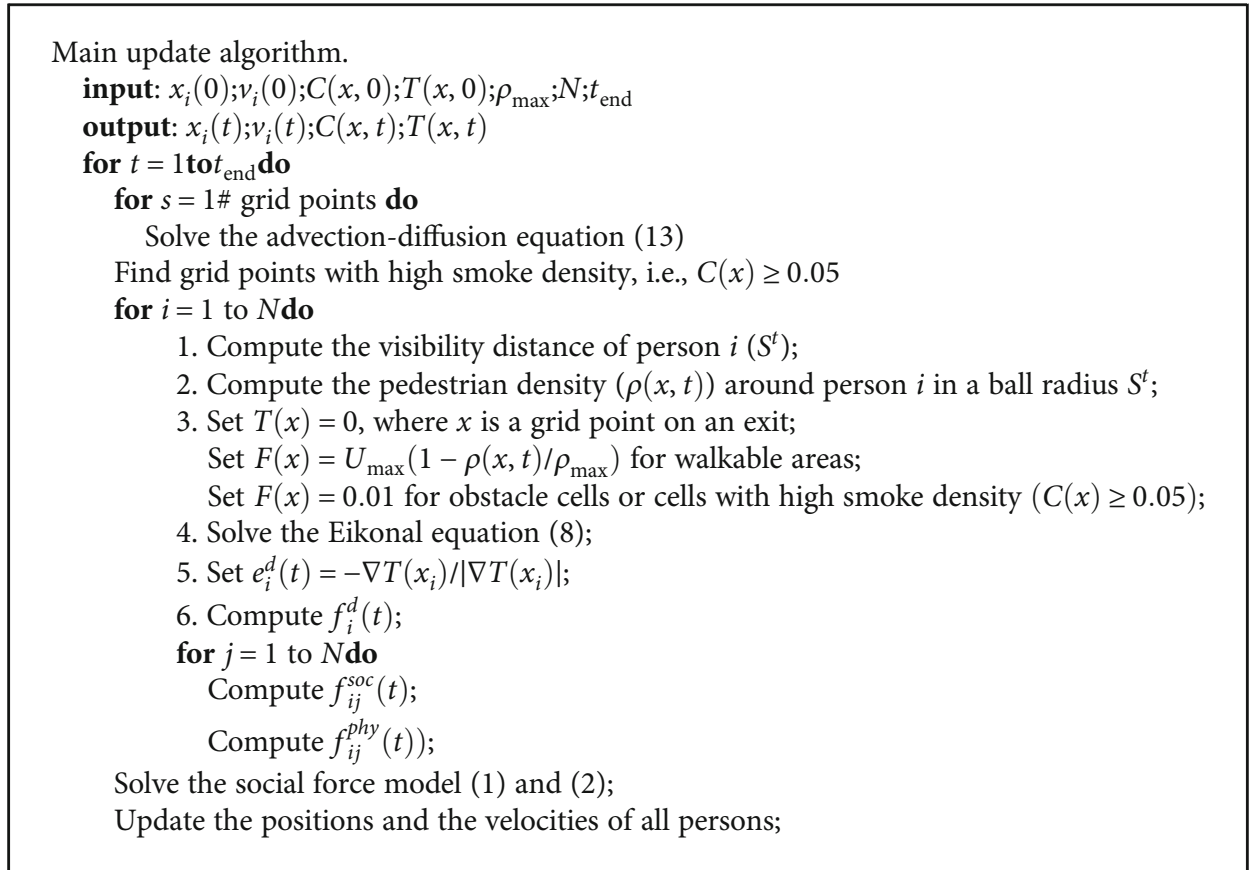

Algorithm 1

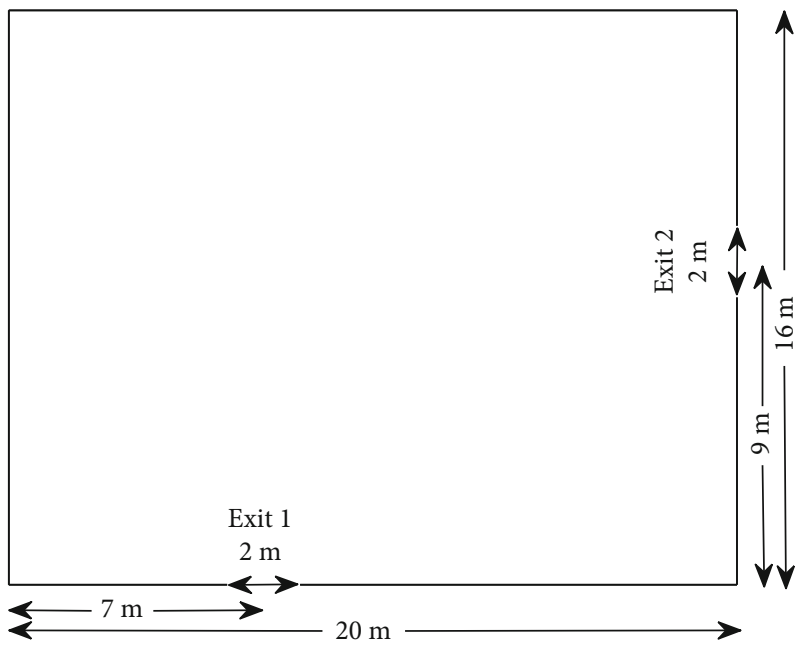

Figure 1: Modeling area for the numerical experiments.

reduces the visibility range of a pedestrian to about $6 \mathrm{~m}-7 \mathrm{~m}$ as described in the references $[13,14] . U$ is the speeddensity function. It describes the relationship between the speed and the density of pedestrians. Presently, there are many speed-density functions available. In our simulation, we choose the following [15]

$$
\begin{gathered}
U(\rho(x, t))=U_{\max }\left(1-\frac{\rho(x, t)}{\rho_{\max }}\right), \\
\rho(x, t)=\frac{1}{\pi\left(S^{t}\right)^{2}} \sum_{j,\left|x-x_{j}\right|<S^{t}} 1 .
\end{gathered}
$$

The term $f_{i j}^{\text {soc }}(t)$ refers to the repulsive social force. It expresses the psychological tendency of pedestrians that prefer to have a personal space and keep a certain safety distance to other pedestrians. The repulsive social force is a monotonic force depending on the interpedestrian distance. It is modeled as an exponential decaying function

$$
f_{i j}^{\mathrm{soc}}(t)=A_{i} \exp \left(\frac{r_{i j}-d_{i j}}{B_{i}}\right) n_{i j}\left(\lambda_{i}+\left(1-\lambda_{i}\right) \frac{1+\cos \left(\varphi_{i j}\right)}{2}\right) .
$$

Here, $A_{i}$ is the interaction strength, $B_{i}$ is the range of the repulsive interactions, and $d_{i j}=\left|x_{i}-x_{j}\right|$ is the distance between the centres of mass of the pedestrians $i$ and $j . r_{i j}=r_{i}$ $+r_{j}$ is the sum of the pedestrians' radii $r_{i}$ and $r_{j}$, and $n_{i j}(t)$ $=\left(n_{i j}^{(1)}, n_{i j}^{(2)}\right)=\left(x_{i}(t)-x_{j}(t)\right) / d_{i j}(t)$ is the normalized vector pointing in the direction from pedestrian $j$ to pedestrian $i$. $\lambda_{i}$ is a value in the range $[0,1]$, and $\lambda_{i}<1$ reflects the behaviour of a pedestrian to react stronger to an event in front of him than to an event behind him. $\cos \left(\varphi_{i j}\right)=-n_{i j}(t) \cdot e_{i}(t)$, where $e_{i}(t)=\left(v_{i}(t)\right) /\left|v_{i}(t)\right|$ is the direction of motion of pedestrian $i$ and $\varphi_{i j}(t)$ denotes the angle between the direction of motion of pedestrian $i\left(e_{i}(t)\right)$ and the direction to pedestrian $j$, which exerts a repulsive force on pedestrian $i$, i.e., $-n_{i j}(t)$.

$f_{i j}^{\mathrm{ph}}(t)$ reflects the physical interaction force. It focuses on the aspect that pedestrians can get in physical contact with each other $\left(r_{i j} \geq d_{i j}\right)$ in crowded environments. This force separates the pedestrians when collisions occur. The physical interaction force is expressed by 
TABLE 1: Parameters for evacuation simulations.

\begin{tabular}{lccc}
\hline Parameter & Symbol & Value & Equation \\
\hline Relaxation time & $\tau_{i}$ & 0.5 & 3 \\
Maximum desired speed & $U^{\max }$ & 10 & $(4)$ \\
Maximum pedestrian density & $\rho^{\max }$ & $A_{i}$ & 0.21 \\
Human-human interaction strength & $B_{i}$ & 0.5 & $(4)$ \\
Human-human range of repulsive interaction & $r_{i j}$ & 0.61 \\
Contact distance & $\lambda_{i}$ & 2 & $(11)$ \\
Anisotropic parameter & $k_{n}$ & 2 \\
Body force coefficient & $k_{t}$ & {$[-0.5,0.5]$} \\
Friction force coefficient & $w_{1}$ & {$[-0.5,0.5]$} \\
Velocity field in $x$-direction & $w_{2}$ & 0.05 \\
Velocity field in $y$-direction & $\kappa_{d}$ & $(11)$ \\
Diffusion constant & $\Delta t$ & 0.02 \\
Time step size & $\Delta x$ & 0.4 \\
Space grid size in $x$ & $\Delta y$ & $(12)$ \\
Space grid size in $y$ & & $(13)$ \\
\hline
\end{tabular}

TABLE 2: Average evacuation time and percentage of pedestrians who move out through Exit 1. The source is in the middle of the room in scenario (i) and in front of Exit 1 in scenario (ii).

\begin{tabular}{lcccc}
\hline $\begin{array}{l}\text { Number of } \\
\text { pedestrians }\end{array}$ & \multicolumn{2}{c}{ Evacuation time (s) } & \multicolumn{2}{c}{ Exit 1 (\%) } \\
& $\begin{array}{c}\text { Scenario } \\
\text { (i) }\end{array}$ & $\begin{array}{c}\text { Scenario } \\
\text { (ii) }\end{array}$ & $\begin{array}{c}\text { Scenario } \\
\text { (i) }\end{array}$ & $\begin{array}{c}\text { Scenario } \\
\text { (ii) }\end{array}$ \\
\hline 100 & $7.4360 \mathrm{~s}$ & $13.3660 \mathrm{~s}$ & 56.70 & 2.60 \\
300 & $13.0800 \mathrm{~s}$ & $22.3920 \mathrm{~s}$ & 170.00 & 4.90 \\
500 & $20.0180 \mathrm{~s}$ & $32.5400 \mathrm{~s}$ & 285.20 & 9.30 \\
\hline
\end{tabular}

$$
f_{i j}^{\mathrm{ph}}(t)=k_{n} H\left(r_{i j}-d_{i j}\right) n_{i j}+k_{t} H\left(r_{i j}-d_{i j}\right) \Delta v_{j i}^{t} t_{i j} \text {, }
$$

where $H$ is the Heaviside function, $t_{i j}=\left(-n_{i j}^{(2)}, n_{i j}^{(1)}\right)$ is the unit tangential vector and orthogonal to $n_{i j}, \Delta v_{j i}^{t}=\left(v_{j}-v_{i}\right)$ - $t_{i j}$ is the tangential velocity difference, and $k_{n}$ and $k_{t}$ are the normal and tangential constants, respectively. The first term on the right-hand side represents a body force for the body compression effect while the second term stands for a sliding friction force that occurs in relative tangential motion.

In the proposed model, the gas density is integrated into the SF model and the Eikonal equation. The development of smoke is expressed through the following linear advectiondiffusion equation [9]

$$
\frac{\partial C}{\partial t}+w \cdot \nabla C=\kappa_{d} \nabla^{2} C+S\left(c_{s}, t\right) \in \Omega \times \mathbb{R}^{+},
$$

with the Dirichlet boundary conditions $C=0$ on $\partial \Omega$, the diffusion constant $\kappa_{d}>0$, the velocity field $w=\left(w_{1}, w_{2}\right) \in \mathbb{R}^{2}$, the source term $S\left(c_{s}, t\right)$, and the simulation domain $\Omega \subset \mathbb{R}^{2}$. We assume that the smoke source emits gas at a constant rate
$Q_{c}[g / s]$ from a single source point $c_{s}=\left(x_{s}, y_{s}\right)$. Hence, we write the source term as

$$
S\left(c_{s}\right)=Q_{c} \delta\left(x-x_{s}\right) \delta\left(y-y_{s}\right)
$$

where $\delta$ is the Dirac delta function given by

$$
\delta(x)=\left(\begin{array}{ll}
1, & x=0 \\
0, & x \neq 0
\end{array}\right.
$$

\section{Numerical Methods}

This section is devoted to numerical methods that are used to discretise the equations of the SF model (1) and (2), the Eikonal equation (8), and the advection-diffusion equation (13).

3.1. Solving the SF Model. To solve the SF model, first, we write the equations (1) and (2) as

$$
u^{\prime}(t)=f(t, u(t)), u\left(t_{0}\right)=u_{0}
$$

with initial condition $u\left(t_{0}\right)=u_{0}$. The equidistant grid $\Omega_{t}$ with respect to time $t$ is generated by

$$
\Omega_{t}=\left\{t_{k}, t_{k}=k \Delta t, k=0,1,2, \cdots, M \text { and } \Delta t=\frac{1}{M}\right\} .
$$

Then, we apply the two-stage second-order Runge-Kutta method as follows

$$
\begin{gathered}
k_{1}=f\left(t_{k}, u_{k}\right), \\
k_{2}=f\left(t_{k}+\frac{2}{3} \Delta t, y_{k}+\frac{2}{3} \Delta t k_{1}\right),
\end{gathered}
$$




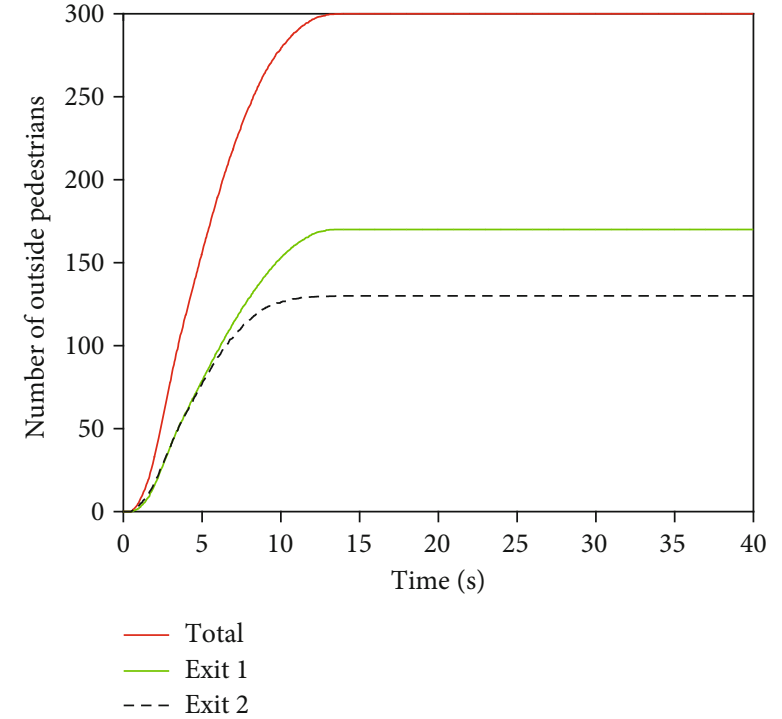

(a) Source is in the middle

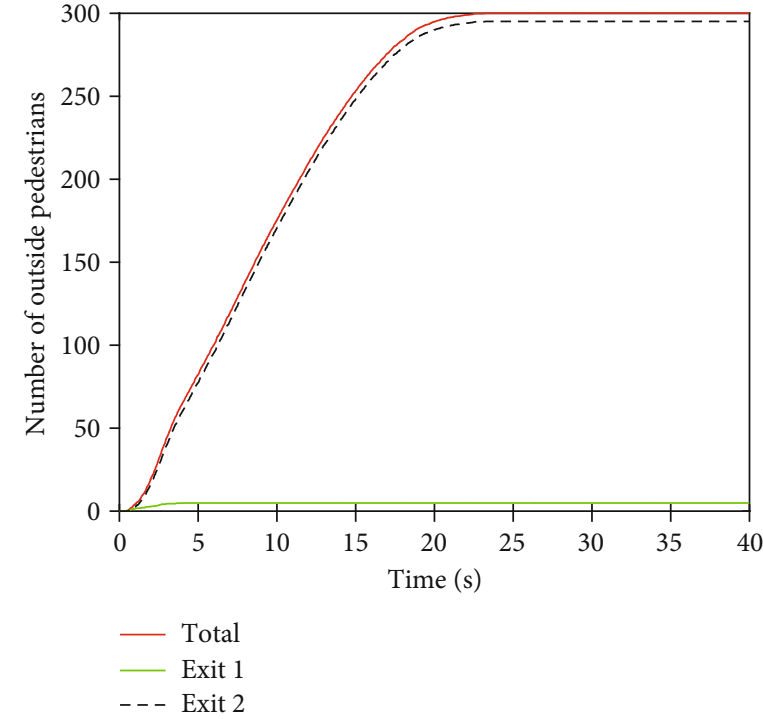

(b) Source is near Exit 1

FIGURE 2: Number of outside pedestrians in scenario (i) and scenario (ii). The total number of pedestrians is 300.

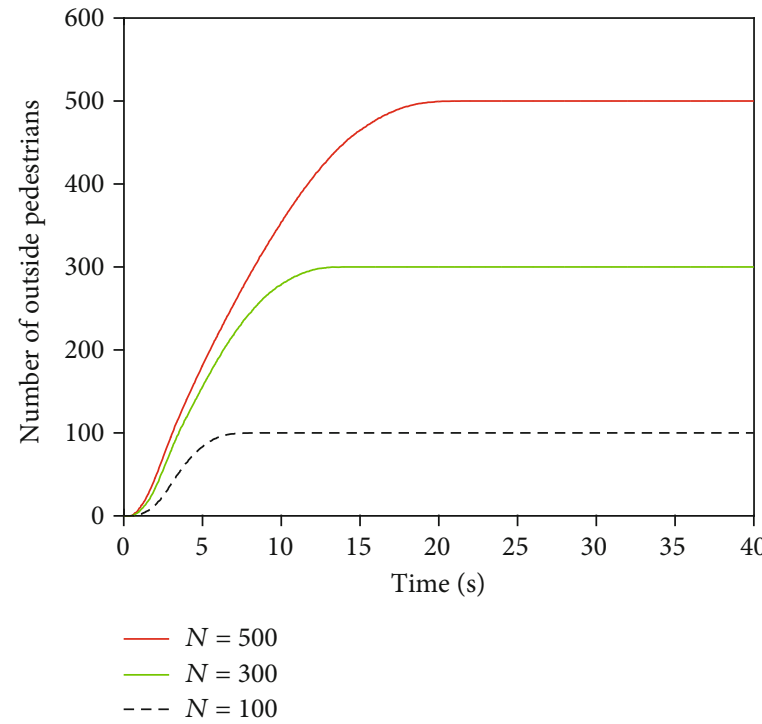

(a) Source is in the middle

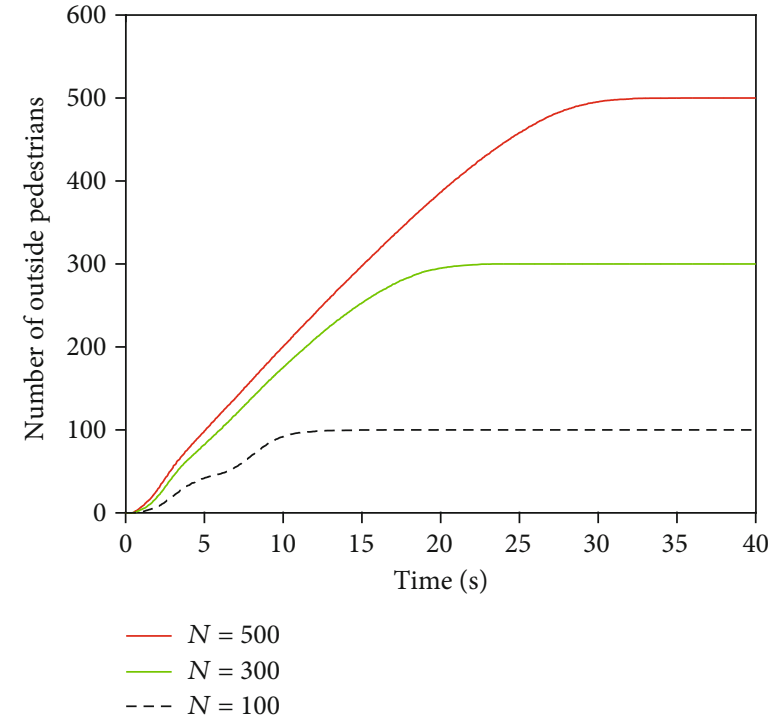

(b) Source is near Exit 1

FIgURE 3: Average number of outside pedestrians in scenario (i) and scenario (ii). The number of pedestrians is 100, 300, and 500.

TABLE 3: The computation time of ten trial runs in scenario (i) and in scenario (ii) with 100,300 , and 500 evacuees.

\begin{tabular}{lcc}
\hline \multirow{2}{*}{ Number of pedestrians } & \multicolumn{2}{c}{ CPU time (10 rounds) } \\
& Scenario (i) & Scenario (ii) \\
\hline 100 & $2.9956 \mathrm{~h}$ & $3.3485 \mathrm{~h}$ \\
300 & $10.6187 \mathrm{~h}$ & $14.8713 \mathrm{~h}$ \\
500 & $22.5628 \mathrm{~h}$ & $34.3668 \mathrm{~h}$ \\
\hline
\end{tabular}

$$
u_{k+1}=u_{k}+\Delta t\left(\frac{1}{4} k_{1}+\frac{3}{4} k_{2}\right) \text {, }
$$

where $u_{k}=u\left(t_{k}\right)$ and $u_{k+1}=u\left(t_{k+1}\right)$. The solution of $u$ in the next time step is obtained from equation (20).

3.2. Solving the Eikonal Equation. There are a quite number of numerical methods existing to approximate the solution of the Eikonal equation: the fast marching method [10], the fast marching level set method [10], the fast sweeping method [16], and the fast iterative method [17] for example. In our experiments, the fast marching method is employed in 


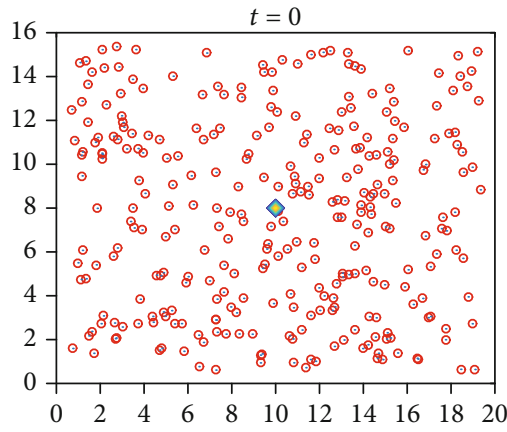

(a)

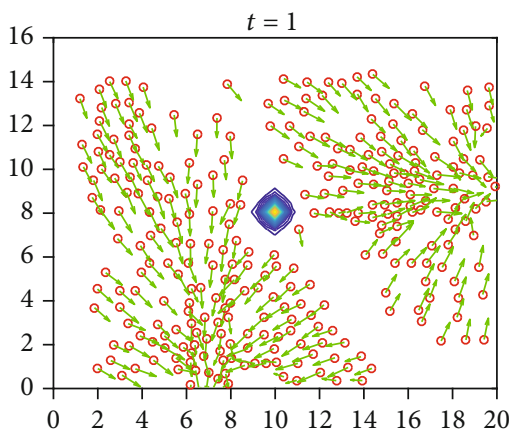

(c)

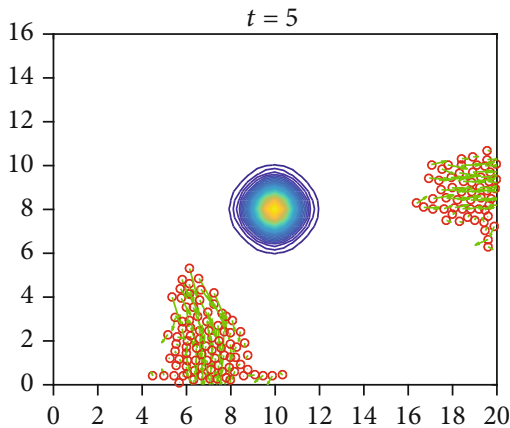

(e)

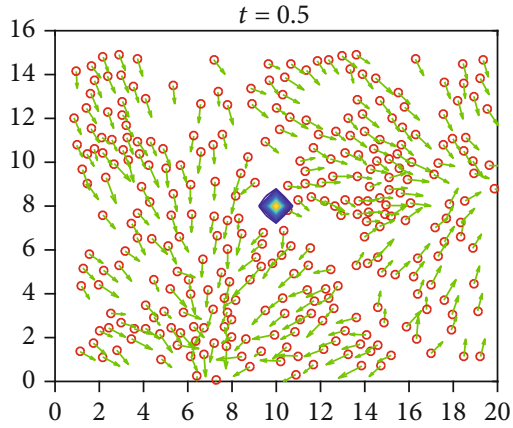

(b)

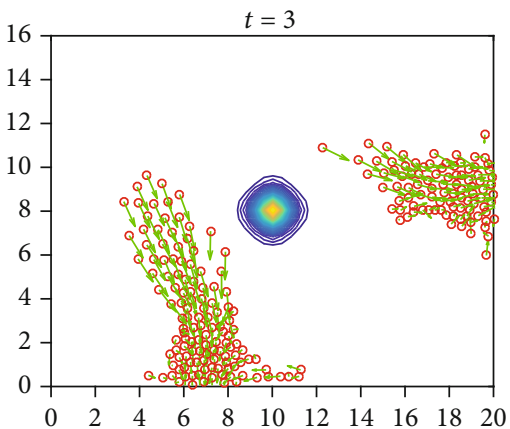

(d)

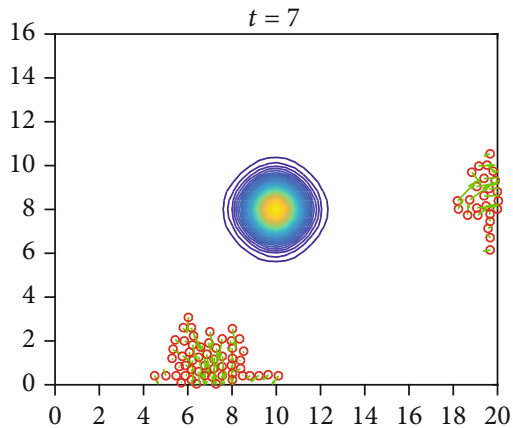

(f)

Figure 4: Movements of 300 pedestrians during smoke spreading, where the source is in the middle of the room. The velocity field $\left(w_{1}, w_{2}\right)$ of the advection-diffusion equation (13) is chosen randomly out of the interval $[-0.5,0.5]$.

all simulations. The details of this method can be reviewed in reference [10].

3.3. Solving the Advection-Diffusion Equation. We discretise the equation of the advection-diffusion (13) in order to obtain the smoke density on the simulation domain at each time step. Equidistant grids with respect to time and space are generated as follows

$$
\begin{gathered}
t_{k}=k \Delta t, k=0,1,2, \cdots, M, \Delta t=\frac{1}{M}, \\
x_{a}=a \Delta x, a=0,1,2, \cdots, N_{x}, \Delta x=\frac{1}{N_{x}},
\end{gathered}
$$

$$
y_{b}=b \Delta y, b=0,1,2, \cdots, N_{y}, \Delta y=\frac{1}{N_{y}} .
$$

Then, we perform the operator splitting on the twodimensional advection-diffusion equation. The $x$-direction and the $y$-direction are solved separately over two time steps. The splitting method on equation (13) reads as

$$
\begin{gathered}
C_{a, b}^{*}=C_{a, b}^{k}-\Delta t\left[w_{1}^{*} D_{x}^{-}\left(C^{*}\right)-\kappa_{d} D_{x x}\left(C^{*}\right)\right] \\
C_{a, b}^{k+1}=C_{a, b}^{*}-\Delta t\left[w_{2}^{t+1} D_{y}^{-}\left(C^{t+1}\right)-\kappa_{d} D_{y y}\left(C^{t+1}\right)\right]+\Delta t S_{a, b}^{k},
\end{gathered}
$$




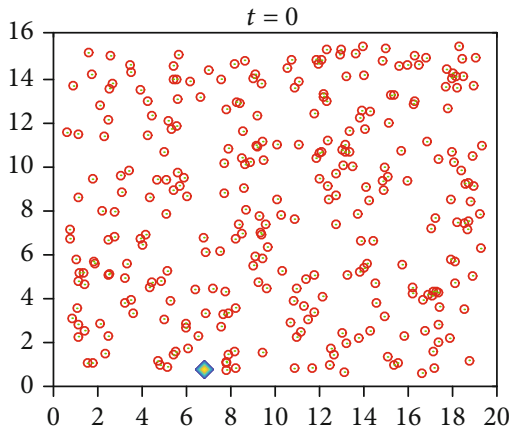

(a)

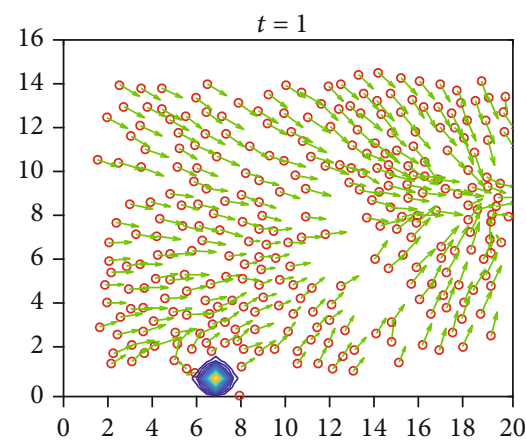

(c)

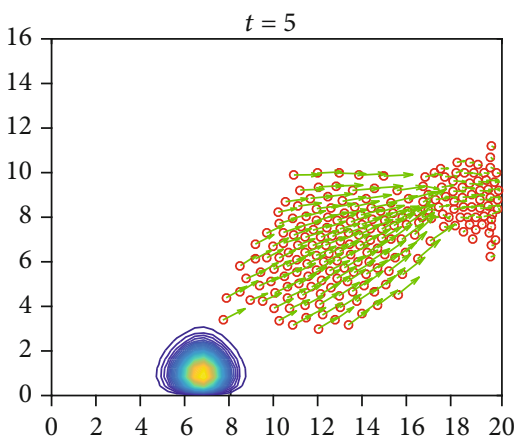

(e)

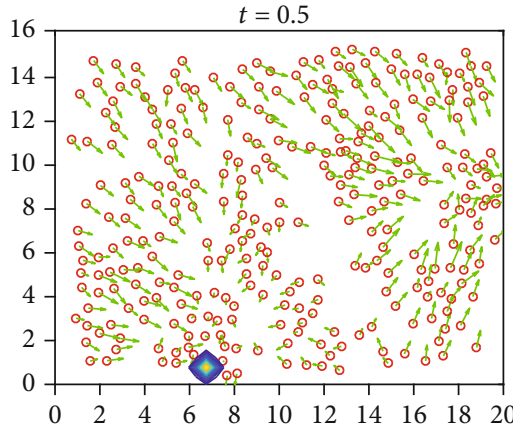

(b)

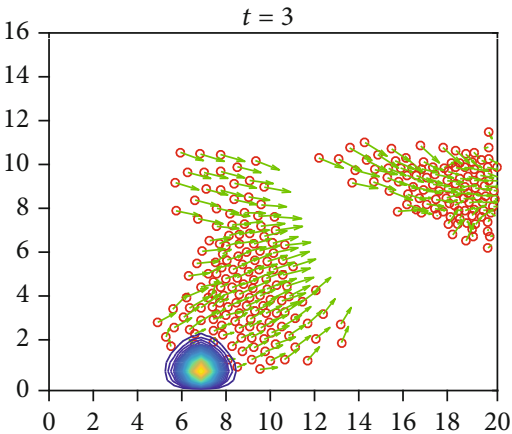

(d)

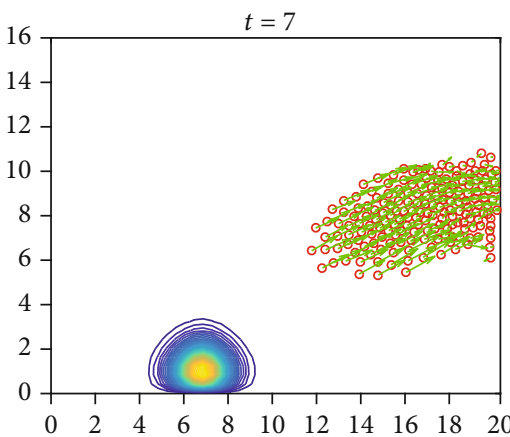

(f)

FIGURE 5: The movements of the pedestrians during smoke spreading, where the source is at Exit 1 . The velocity field $\left(w_{1}, w_{2}\right)$ of the advection-diffusion equation (13) is chosen randomly out of the interval $[-0.5,0.5]$.

where

$$
\begin{gathered}
D_{x}^{-}\left(C^{*}\right)=\frac{C_{a, b}^{*}-C_{a-1, b}^{*}}{\Delta x}, \\
D_{x x}\left(C^{*}\right)=\frac{C_{a+1, b}^{*}-2 C_{a, b}^{*}+C_{a-1, b}^{*}}{\Delta x^{2}}, \\
D_{y}^{-}\left(C^{k+1}\right)=\frac{C_{a, b}^{k+1}-C_{a, b-1}^{k+1}}{\Delta y}, \\
D_{y y}\left(C^{k+1}\right)=\frac{C_{a, b+1}^{k+1}-2 C_{a, b}^{k+1}+C_{a, b-1}^{k+1}}{\Delta y^{2}} .
\end{gathered}
$$

We assume that the smoke density on the boundaries is zero. Thus, we have

$$
C(0, y, t)=0, C\left(N_{x}, y, t\right)=0, C(x, 0, t)=0, C\left(x, N_{y}, t\right)=0,
$$

with the initial condition given by

$$
C(x, y, 0)=Q_{c} \delta\left(x-x_{s}\right) \delta\left(y-y_{s}\right),
$$

where $\left(x_{s}, y_{s}\right)$ is a hazard source point and $Q_{c}$ is the gas concentration at the initial time. First, we solve implicitly equation (24) in the $x$ direction and obtain $C_{a, b}^{*}$ for all grid points $a, b$. The value $C_{a, b}^{*}$ is used for equation (25) in $y$ direction. Equation (3.6) is solved implicitly. Finally, we receive the smoke density for the next time step $C_{a, b}^{k+1}$ for all grid points $a, b$. For further details and the convergence of this method, we refer to reference [18]. 


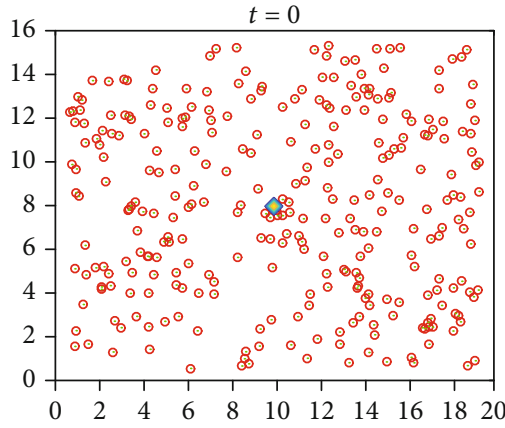

(a)

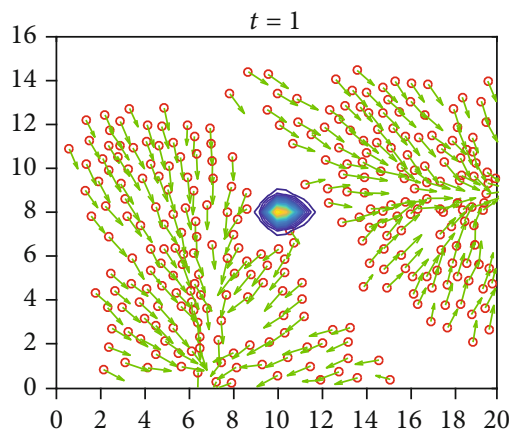

(c)

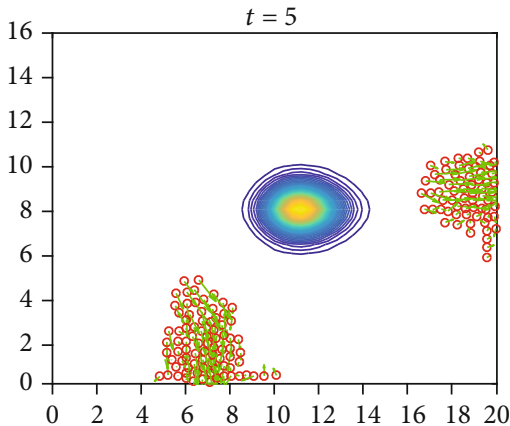

(e)

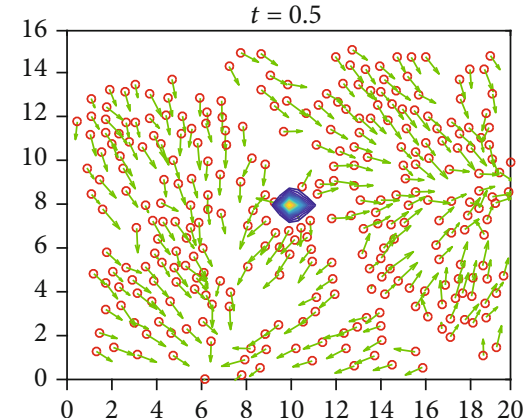

(b)

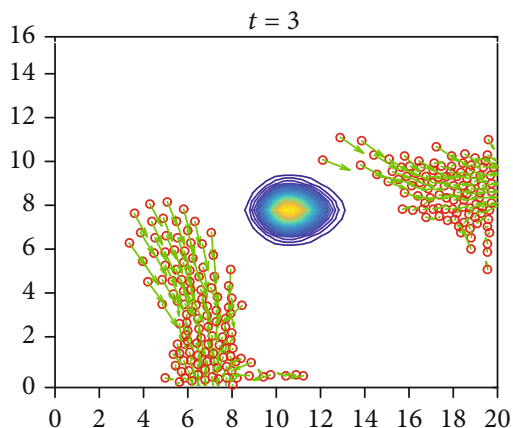

(d)

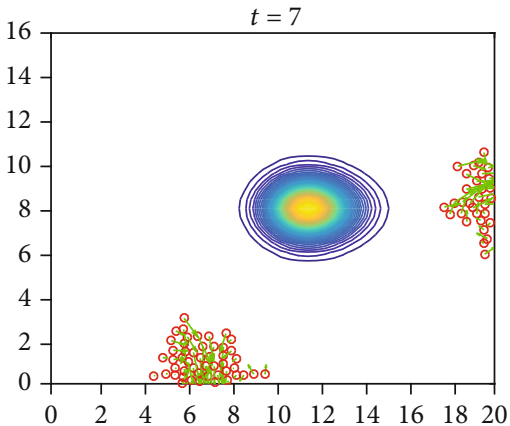

(f)

FIGURE 6: The movements of 300 pedestrians during smoke spreading, as the velocity field of the advection-diffusion is $\left(w_{1}, w_{2}\right)=(0.5,0)$ in equation (13) and the source is in the middle of the room.

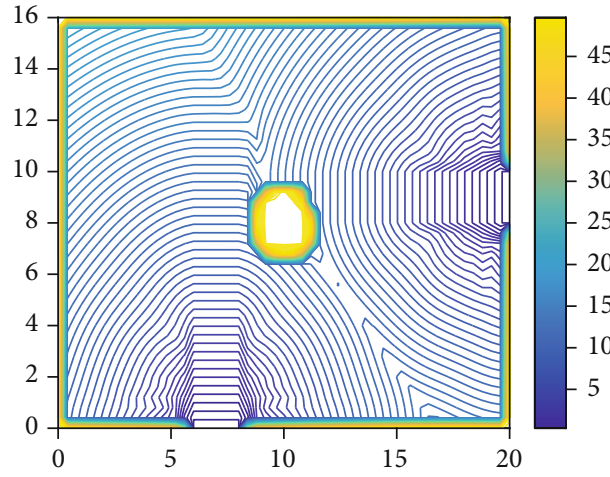

(a) Scenario (i)

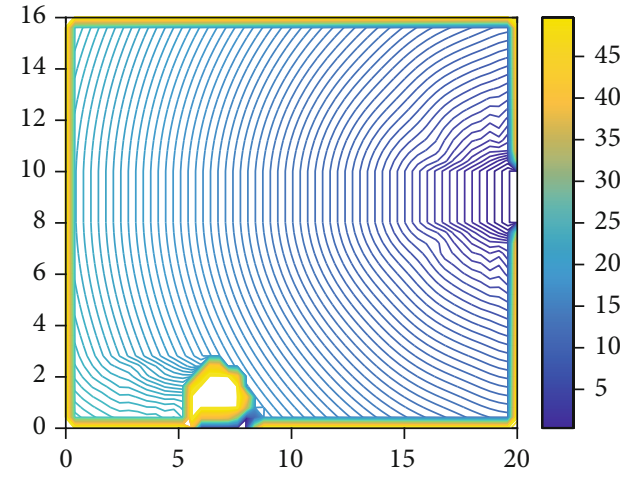

(b) Scenario (ii)

Figure 7: Contour plots of the dynamic potential (estimated remaining travel time) of 300 pedestrians in scenario (i) and scenario (ii) at $t=5 \mathrm{~s}$. 


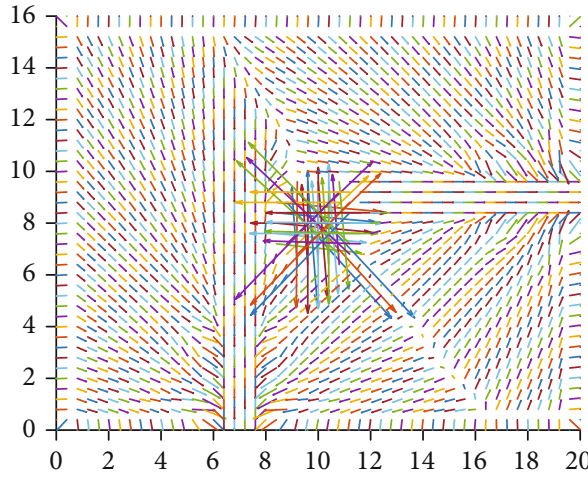

(a) Scenario (i)

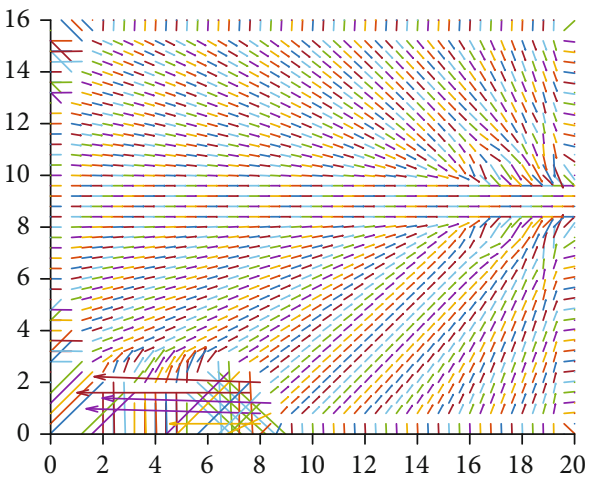

(b) Scenario (ii)

FIGURE 8: Negative gradient of the arrival times of 300 pedestrians in scenario (i) and scenario (ii) at $t=5 \mathrm{~s}$.

3.4. Main Update Algorithm. A way to obtain an individuals' trajectory in each time step is described in Algorithm 1. The algorithm, that is used to update an individuals' position and velocity in each time step, is shown below.

Remark 1. $>$ We set the threshold smoke concentration as $C$ $(x) \geq 0.05$ which is assumed to be high enough to influence the movements of the pedestrians. This value reduces the visibility range of a pedestrian to about $6 \mathrm{~m}-7 \mathrm{~m}$, and his speed is affected significantly, as described in the references $[13,14]$.

\section{Numerical Experiments and Results}

In this section, we investigate numerical experiments which are performed in a room of size $16 \mathrm{~m} \times 20 \mathrm{~m}$. Two exits of width $2 \mathrm{~m}$ are located at the bottom and on the right side of the room. They are labeled as Exit 1 and Exit 2, respectively, see Figure 1. Since our model is nondeterministic, different runs will present different results. Thus, to obtain more general results, we simulate ten trial runs for the same example and their average is used. The computations are executed on a HP Intel(R) Core(TM) i7-7700CPU, 3.6GHz. All programs are implemented in MATLAB.

To examine the effect of smoke spreading on an individual's movements, we limit our study to only one source and assume that the smoke density at the source point is rather high at the initial time. Furthermore, the source emits a constant smoke density of $0.01 \mathrm{~g} / \mathrm{s}$ afterward, i.e.,

$$
Q_{c}=\left(\begin{array}{ll}
10 \mathrm{~g} / \mathrm{s} & , t=0 \\
0.01 \mathrm{~g} / \mathrm{s} & , t>0 .
\end{array}\right.
$$

The velocity field $\left(w_{1}, w_{2}\right)$ in equation (13) is chosen randomly out of the interval $[-0.5,0.5]$ at each time step. The maximum velocity $U_{\max }$ is set to $3 \mathrm{~m} / \mathrm{s}$, and the maximum density $\rho_{\max }$ is $10 \mathrm{ped} / \mathrm{m}^{2}$. At initial time, the pedestrians are randomly distributed in the room, and their velocities are set to zero.

The model parameters that are used in the evacuation simulations are shown in Table 1 . We simulate the individuals' temptation to move away from a high smoke density area and to simultaneously move towards an exit to evacuate. Two scenarios are regarded to examine the influence of the smoke source position on the pedestrian evacuation. In scenario (i), the smoke source's location is in the middle of the room, whereas it is located in front of Exit 1 in scenario (ii). There are 100, 300, and 500 individuals in experiments.

The average evacuation time and the percentage of pedestrians who move out through Exit 1 in scenario (i) and in scenario (ii) are presented in Table 2. We see that the average evacuation time in scenario (ii) is longer than in scenario (i) in all cases. Therefore, the location of the smoke source affects the pedestrian's evacuation time. It takes more time to evacuate when the source is placed in front of Exit 1 compared to when the source is in the middle of the room. A high number of evacuees move out through Exit 2 in scenario (ii) leading to a large jamming and crowd around this exit. In scenario (ii), the pedestrians reject to move out through Exit 1 because the source is in front of this exit and it is too dangerous to move out.

The plot of the average number of outside pedestrians of each exit versus the time in scenario (i) and scenario (ii) with 300 individuals are demonstrated in Figure 2. The usage of Exit 1 is slightly higher than the usage of Exit 2 in scenario (i), in which the smoke source is placed in the center of the room. The pedestrians evacuate less out of Exit 1 in scenario (ii).

The comparisons of the average number of outside pedestrians in scenario (i) and scenario (ii) with 100, 300, and 500 pedestrians in the experiments are displayed in Figure 3. Both scenarios show that as the number of pedestrians in experiments rises, the average number of outside pedestrians also increases. The time that is needed to evacuate all individuals out of the room increases when the number of pedestrians in simulations get higher. Scenario (ii) takes more time to evacuate all people compared to scenario (i).

Table 3 shows the computation time of ten trial runs in scenario (i) and in scenario (ii) with 100, 300, and 500 pedestrians. In all cases, the simulations in scenario (ii) take more computation time compared to scenario (i). This is because the evacuation times in scenario (ii) are larger than in scenario (i), see Table 2.

Figure 4 demonstrates the movement directions of 300 evacuees during smoke spreading, where the smoke source 
is in the center of the room. Red circles refer to the positions of pedestrians, and green arrows show the moving directions. At time $t=0$, all pedestrians are placed randomly inside the room and their velocities are zero. There is no green arrow points out from any pedestrian. As time goes on, the individuals start to move. The moving direction of each pedestrian points in the direction towards the exits and points out from the smoke region. Clogging and arching effects are observed around the two exits as we can see in Figures 4(d)-4(f). The number of individuals who evacuate through Exit 1 is slightly higher than the number of individuals who move towards Exit 2.

The movements of 300 pedestrians during the spreading of smoke in scenario (ii), where the source is at Exit 1, are illustrated in Figure 5. At the beginning of the simulation, the smoke density is less and has no effect on the individuals' movements. The moving directions of some pedestrians point to Exit 1 where the smoke source is situated. Pedestrians, who are near Exit 1, go out through this exit, see Figure 5(b). While the time increases, the smoke density around Exit 1 increases. The moving directions of the individuals change. They point out of the smoke source region and point mostly towards Exit 2, see Figure 5(c). Most of the pedestrians reject to evacuate through Exit 1 since it is dangerous. Large crowds and jammings occur around Exit 2, see Figure 5(f).

The trajectories of the 300 evacuees during propagation of smoke are presented in Figure 6, in which the smoke source is in the middle of the room and the velocity field of the advection-diffusion is $\left(w_{1}, w_{2}\right)=(0.5,0)$ in equation (13).

Figure 7 displays the contour plot of the traveling time (arrival time) to reach a destination of the 300 individuals in the simulations considering scenario (i) and scenario (ii) at $t=5 \mathrm{~s}$. The traveling cost is obtained by solving the Eikonal equation (8) with the help of the fast marching method. To plot the traveling time, we set $T(x)=50$ for grid space that receives traveling cost higher than $50(T(x)>50)$, such as high smoke density grids or walls grids. In Figure 7 , we see that the arrival times are relatively high in areas where the smoke source or the wall grids are placed. This is on account of combining the smoke density and obstacles to the speed of the front $(F(x))$ in equation (2.6).

Negative gradients of the arrival times $(-\nabla T(x) /|\nabla T(x)|)$ of 300 individuals for the example in scenario (i) and scenario (ii) at $t=5 \mathrm{~s}$ are shown in Figure 8. They demonstrate the movement directions at each point on the simulation domain which guides the way to move for the evacuees. In Figure 8 , we see that negative gradients of the arrival times point out of the smoke source and wall areas. They point towards the exits.

\section{Discussion and Conclusions}

In this paper, we present a model that is used to describe pedestrians' movements during the propagation of smoke. The social force model is applied and coupled with the advection-diffusion equation and the Eikonal equation. The advection-diffusion equation is employed for smoke spreading. The Eikonal equation is used for the moving directions of pedestrians. It depends on the smoke as well as on the pedestrian density in the visibility range. In our model, we observed the arching and the clogging effect which are pedestrian collective phenomena occurring in pedestrian crowds around exits [2, 19]. We performed different experiments and showed their results.

\section{Data Availability}

Previously reported data were used to support this study and are cited at relevant places within the text as references $[2,19]$.

\section{Conflicts of Interest}

The author(s) declare(s) that they have no conflicts of interest.

\section{Acknowledgments}

This research is supported by the Department of Mathematics, Science Faculty at Kasetsart University, Thailand. The author is very grateful for the working environments provided by the Department of Mathematics at Kasetsart University, Thailand. I would like to thank Markus Guth for valuable and inspiring comments.

\section{References}

[1] D. Helbing, "A fluid-dynamic model for the movement of pedestrians," Complex Systems, vol. 6, pp. 391-415, 1992.

[2] D. Helbing, I. J. Farkas, P. Molnár, and T. Vicsek, Simulation of Pedestrian Crowds in Normal and Evacuation Situations, Pedestrian and Evacuation Dynamics, Springer, Berlin, Germany, 2002.

[3] A Nakayama and Sugiyama, "Two-dimensional optimal velocity model for pedestrians and biological motion," AIP Conference Proceedings, vol. 661, p. 107, 2003.

[4] S. Okazaki, "A study of simulation model for pedestrian movement with evacuation and queuing, Part 1: Pedestrian Movement by the Application on of Magnetic Models," Trans. of A.I.J., vol. 35, no. 283, pp. 111-119, 1979.

[5] J. Makmul, “A cellular automaton model for pedestrians' movements influenced by gaseous hazardous material spreading," Modelling and Simulation in Engineering, 10 pages, 2020.

[6] G. Antonini, M. Bierlaire, and M. Weber, "Discrete choice models of pedestrian walking behavior," Transportation Research Part B, vol. 40, no. 8, pp. 667-687, 2006.

[7] Y. Zheng, X. Li, N. Zhu, B. Jia, and R. Jiang, "Evacuation dynamics with smoking diffusion in three dimension based on an extended floor-field model," Physica A, vol. 507, pp. 414-426, 2018.

[8] W. Yuan and K. H. Tan, "A model for simulation of crowd behaviour in the evacuation from a smoke-filled compartment," Physica A, vol. 390, no. 23-24, pp. 4210-4218, 2011.

[9] J. M. Stockie, "The mathematics of atmospheric dispersion modelling," Society for Industrial and Applied Mathematics, vol. 53, no. 2, pp. 349-372, 2011.

[10] J. A. Sethian, "Advancing Interfaces: Level Set and Fast Marching Methods," in Proceedings of the National Acadenamic of Sciences, 1995. 
[11] T. Kretz, A. Groess, S. Hengst, L. Kautzsch, A. Pohlmann, and P. Vortisch, "Quickest paths in simulations of pedestrians," Advances in Complex Systems, vol. 14, no. 5, pp. 733-759, 2011.

[12] G. A. Frank and C. O. Dorso, "Evacuation under limited visibility, International," Journal of Modern Physics, vol. 26, no. $1,2015$.

[13] S. C. Cao, W. G. Song, X. D. Liu, and N. Mu, "Simulation of pedestrian evacuation in a room under fire emergency," Procedia Engineering, vol. 71, pp. 403-409, 2014.

[14] G. E. Mulholland, "Smoke production and properties," The SFPE Handbook of Fire Protection Engineering, pp. 217-227, Springer, 1995, second ed.

[15] B. Piccoli and A. Tosin, "A review of continuum mathematical models of vehicular traffic," Encyclopedia of Complexity and Systems Science, vol. 9727-9749, pp. 1-33, 2009.

[16] P. A. Gremaud and C. M. Kuster, "Computational study of fast methods for the eikonal equation," SIAM Journal on Scientific Computing, vol. 27, no. 6, pp. 1803-1816, 2006.

[17] W. K. Jeong and R. T. Whitaker, A Fast Eikonal Equation Solver for Parallel System, 2007, SIAM conference on Computational Science and Engineering.

[18] J. Makmul, Microscopic and macroscopic for pedestrian crowds, [Phd thesis], Mannheim University, 2016.

[19] D. Helbing and T. Vicsek, "Simulating Dynamical Features of Escape Panic,” Nature, vol. 407, no. 6803, pp. 487-490, 2000. 